\title{
Abnormalities in exercising skeletal muscle in congestive heart failure can be explained in terms of decreased mitochondrial ATP synthesis, reduced metabolic efficiency, and increased glycogenolysis
}

Graham J Kemp, Campbell H Thompson, John R Stratton, Francois Brunotte, Michael Conway, Stamatis Adamopoulos, Leonard Arnolda, George K Radda, Bheeshma Rajagopalan

\begin{abstract}
Objective-To distinguish between the effects of reduced oxidative capacity and reduced metabolic efficiency on skeletal muscle bioenergetics during exercise in patients with congestive heart failure.

Design and patients-Patients were studied by ${ }^{31} \mathbf{P}$ magnetic resonance spectroscopy during aerobic exercise and recovery, and results compared with controls.

Results-In flexor digitorum superficialis muscle (26 patients) there was a $30 \%$ decrease in oxidative capacity compared with control (mean (SE) 36 (2) $v 51$ (4) $\mathrm{mM} / \mathrm{min}$ ) and also a $40 \%$ decrease in "effective muscle mass" (5 (1) $v 9$ (1) arbitrary units), probably at least partly the result of reduced metabolic efficiency. Both contribute to increased phosphocreatine depletion and intracellular acidosis during exercise. However, an increased concentration of ADP (an important mitochondrial regulator) during exercise permitted near-normal rates of oxidative ATP synthesis. Results were similar in gastrocnemius muscle (20 patients), with a $30 \%$ decrease in maximum oxidative capacity (29 (4) $v 39(3) \mathrm{mM} / \mathrm{min})$ and a $65 \%$ decrease in effective muscle mass (5 (1) $v 13$ (2) arbitrary units). Exercise training improved maximum oxidative capacity in both muscles, and in gastrocnemius effective muscle mass also.

Conclusions-Skeletal muscle exercise abnormalities in patients with congestive heart failure result more from decreased metabolic efficiency than from the abnormalities in mitochondrial oxidation. Both decreased efficiency and defective mitochondrial oxidation result in an increased activation of glycogen phosphorylase, and may be improved by exercise training.
\end{abstract}

(Heart 1996;76:35-41)

Keywords: bioenergetics; congestive heart failure; ${ }^{31} \mathrm{P}$ magnetic resonance spectroscopy; skeletal muscle

Congestive heart failure is associated with a reduction in exercise tolerance, which is thought to be largely attributable to abnormal function of skeletal muscle. ${ }^{31} \mathrm{P}$ magnetic reso- nance spectroscopy, which provides a means to study muscle bioenergetics in vivo, has been used to show that heart failure is associated with increased phosphocreatine breakdown and intracellular acidosis during exercise in human leg muscle, ${ }^{1-3}$ human forearm muscle, ${ }^{45}$ and rat leg muscle. ${ }^{67}$ In general, ${ }^{8}$ these could result from a defect in oxidative ATP synthesis, an increase in the requirements for ATP due to reduced muscle mass and/or metabolic efficiency, or a primary over-activation of glycogenolysis. The first of these mechanisms is supported by the slow phosphocreatine recovery after exercise (a purely oxidative process) in heart failure ${ }^{2910}$ as well as by direct measurements of oxygen consumption during exercise, ${ }^{11}$ and could result from either inadequate vascular oxygen supply or of a reduction in the number (or capacity) of mitochondria, and there is some evidence for both. Blood flow is low during heavy exercise protocols, ${ }^{12} 13$ although metabolic abnormalities are also observed during moderate exercise, despite normal blood flow and ventilation. ${ }^{14}{ }^{14}$ Recent studies of the adequacy of muscle blood flow in heart failure using measurements of haemoglobin/myoglobin desaturation have produced conflicting results. ${ }^{1516}$ Mitochondrial numbers $^{12}$ and muscle oxidative enzymes ${ }^{2617}$ are reduced in congestive heart failure. However, abnormalities are seen in ischaemic exercise, ${ }^{18} 19$ in which oxidative ATP synthesis is negligible, and though part of these abnormalities may be due to muscle fibre atrophy, ${ }^{20}$ this is probably not a complete explanation. ${ }^{2419}$ This suggests that there is a decrease in metabolic efficiency, perhaps the result of fibre type change. ${ }^{172021}$

In this paper we use current methods of analysis $^{8}$ to separate the contributions of some of these mechanisms to the ${ }^{31} \mathrm{P}$ magnetic resonance spectroscopic abnormalities observed during mixed aerobic exercise in an arm and a leg muscle in congestive heart failure, and also to study the changes produced by exercise training. Some of the basic ${ }^{31} \mathrm{P}$ magnetic resonance spectroscopy data, but not the present analysis, have been presented separately. ${ }^{1} 92223$

\section{Patients and methods}

PATIENTS

Patients were in stable chronic heart failure (no recent myocardial infarction, coronary artery bypass grafting, or change in medica-

\author{
$\mathrm{L}_{\mathrm{MAX}}=$ apparent maximum \\ rate of glycogenolytic ATP \\ synthesis; \\ $\mathrm{PCr}=$ phosphocreatine; \\ $\mathrm{Pi}=$ inorganic phosphate \\ 6 February 1996 \\ Symbols and \\ abbreviations
$\mathrm{Q}_{\operatorname{MAX}}=$ apparent maximum
}


tions). Diagnosis was based on standard clinical, laboratory, radiological, and electrocardiographic criteria.

Studies of the forearm finger flexor muscle (flexor digitorum superficialis) were performed on 26 male patients, aged 42-78 (mean 58 ) years. The mean duration of heart failure was 3.5 years (range $0.4-11$ years). Six patients were in New York Heart Association class I (five patients with coronary artery disease, one with dilated cardiomyopathy); 14 patients were in class II (nine patients with coronary artery disease, two with valvar heart disease, three with idiopathic cardiomyopathy); one patient with coronary artery disease was in class III; and five patients were in class IV (four patients with coronary artery disease, one with idiopathic cardiomyopathy). Mean (SD) body mass was 71 (10) $\mathrm{kg}$ in class I, 78 (5) $\mathrm{kg}$ in class II, and 86 (22) $\mathrm{kg}$ in class III and IV combined. Mean (SD) ejection fraction measured by radionuclide angiography or by cross sectional echocardiography was 28 (11)\% (measured in 5/6 patients) in class I, 21 (12)\% (measured in $8 / 14$ patients) in class II, and $20(2) \%$ (measured in $6 / 6$ patients) in class III and IV. All patients were taking diuretic drugs, 16 were taking angiotensin converting enzyme inhibitor drugs, five were taking amiodarone, three were taking calciumchannel antagonist drugs, three were taking $\beta$ receptor antagonist drugs, and two were taking digoxin. Training studies were performed on a subgroup of 10 patients, aged 42-78 (mean 62) years. The characteristics of this group and details of the one month isometric/isotonic training protocol have been described in detail elsewhere. ${ }^{22}$

Studies of the calf muscle (gastrocnemius) were performed on 20 male patients, aged 43-75 (mean 63) years. The mean duration of heart failure was 2.0 years (range $0.5-9$ years). Twelve patients were in class II (10 patients with coronary artery disease, one with valvar heart disease, one with idiopathic cardiomyopathy); eight patients were in class III (seven patients with coronary artery disease, one with idiopathic cardiomyopathy). Mean (SD) body mass was 81 (13) kg in class II and $72(10) \mathrm{kg}$ in class III. Mean (SD) ejection fraction was $29(8) \%$ (measured in 10/12 patients) in class II and 12 (3)\% (measured in 6/8 patients) in class III.

Of all the patients, 19 were taking diuretic drugs, 18 were taking angiotensin converting enzyme inhibitor drugs, three were taking amiodarone, one was taking a calcium-channel antagonist drug, and five were taking digoxin. Six of the patients also had forearm studies. Training studies were performed on a subgroup of 12 patients, aged 43-75 (mean 62) years. The characteristics of this group and details of the two month bicycle ergometer training protocol have been described in detail elsewhere. ${ }^{9}$

Results from the forearm were compared with those of 22 healthy male controls with similar age-range (34-71 years) and mean age (55 years) to the patient group. Results from the calf were compared with those of 15 healthy male controls (33-68, mean 55 years). None had any evidence of cardiac disease, diabetes, or hypertension. All subjects gave informed consent according to a protocol approved by the local hospital ethics committee.

\section{MAGNETIC RESONANCE SPECTROSCOPY}

METHODS

For forearm studies, the dominant arm was placed in a 1.9 Tesla superconducting magnet (Oxford Instruments, Oxford) interfaced to a Biospec spectrometer (Oxford Research Systems, Oxford) and a $2.5 \mathrm{~cm}$ diameter surface coil was placed over the muscle. Spectra were acquired using a $2 \mathrm{~s}$ interpulse delay at rest (128 scans) and during finger flexion (32 scans) at a power output of $0.25 \mathrm{~W}$ for four spectra, increased by $0.08 \mathrm{~W}$ for each of the remaining spectra. Exercise was continued until fatigue. The muscle was then studied for 12 minutes during recovery (four 16-scan spectra, four 32-scan spectra, and then two 64-scan spectra).22 For calf muscle studies, subjects were placed in a 2.0 Tesla superconducting magnet (Oxford Magnet Technology, Eynsham, Oxford) interfaced to a Bruker spectrometer (Bruker, Coventry) with the right calf overlying a $6.0 \mathrm{~cm}$ diameter surface coil. Spectra were acquired using a $2 \mathrm{~s}$ interpulse delay at rest (128 scans) and during plantar flexion (32 scans) at a power output of $1.5 \mathrm{~W}$ for four spectra, increased by $0.5 \mathrm{~W}$ for each of the remaining spectra. Exercise was continued until fatigue. The muscle was then studied for 12 minutes during recovery (four 16 -scan spectra then eight 32 -scan spectra). ${ }^{9}$

\section{DATA ANALYSIS}

Spectra were analysed as described before ${ }^{24}$ : cytosolic $\mathrm{pH}$ was obtained from the chemical shift of inorganic phosphate $(\mathrm{Pi})$; the concentrations of phosphocreatine (PCr) and inorganic phosphate ( $\mathrm{mM}$, that is, $\mathrm{mmol} / \mathrm{l}$ cytosolic water) were obtained from the saturation-corrected metabolite ratios assuming an ATP concentration of $8.2 \mathrm{mM}$; and free cytosolic ADP concentration was calculated from $\mathrm{pH}$ and phosphocreatine concentration assuming the creatine kinase equilibrium. (During exercise it is convenient to express phosphocreatine concentration as $\mathrm{PCr} /(\mathrm{PCr}+$ $\mathrm{Pi})$ to allow for changes in signal intensity due to possible movement.) The details of the kinetic analysis of the data have been published elsewhere. ${ }^{82526}$ Briefly, an analysis of recovery from exercise is used to calculate both the halftime of phosphocreatine recovery, which is an inverse measure of mitochondrial function, and the apparent maximum rate of oxidative ATP synthesis $\left(Q_{\operatorname{MAx}}\right)$, which is a quantitative measure of mitochondrial capacity (a function of mitochondrial content, mitochondrial activation state, and blood flow). The kinetics of $\mathrm{pH}$ recovery after exercise reflect net proton efflux, whose absolute rate and $\mathrm{pH}$-dependence can be assessed by quantitative analysis. During exercise, ATP production by net hydrolysis of phosphocreatine is measured directly, while measurements of 

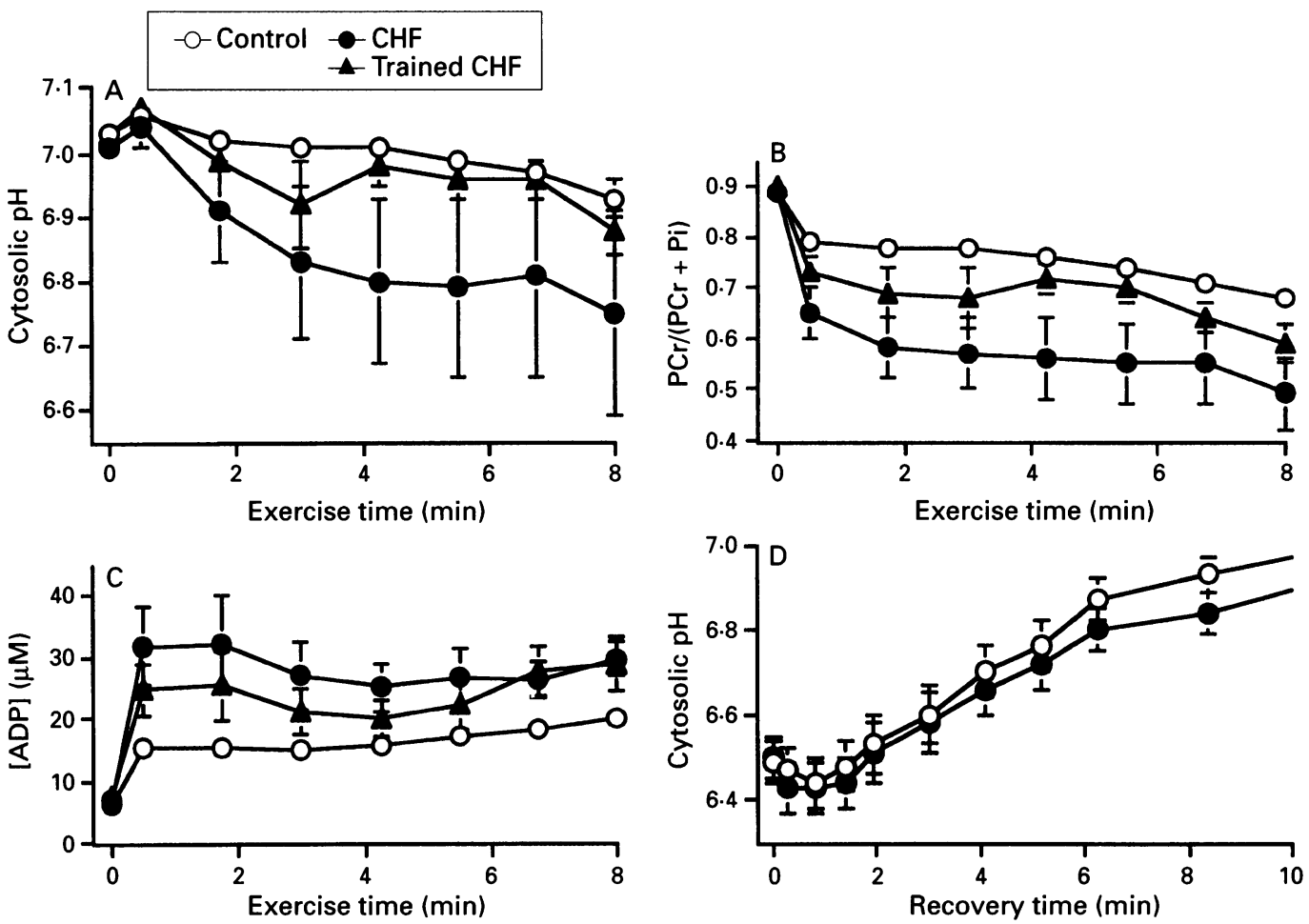

Figure 1 Metabolite changes during exercise and recovery in flexor digitorum superficialis. The first three panels show the time course of $(A)$ cytosolic $p H,(B) P C r /(P C r+P i)$, and $(C)$ free cytosolic ADP concentration during 8 exercise spectra in all the untrained patients (closed circles) and controls (open circles), and also in trained patients (triangles). Later during exercise the number of subjects contributing to each point decreased because some, particularly patients, had already stopped exercising. Results were significantly different from control for all three variables $(P<0.03)$. (D) The time course of cytosolic $\mathrm{pH}$ during recovery in all (untrained) congestive heart failure patients (closed circles) and controls (open circles). All results are given as mean (SE).

$\mathrm{pH}$ and phosphocreatine concentration are used to calculate the rate of glycogenolytic ATP synthesis, after appropriate correction for net proton efflux. In particular, the initial rate of nonoxidative ATP synthesis is used to calculate the effective muscle mass, which is the product of metabolic efficiency and cross sectional area. In later exercise, oxidative ATP synthesis is calculated by difference. As this is largely under the control of free ADP concentration, we use the ADP concentration and the oxidative ATP synthesis rate to calculate the time-course of apparent $\mathbf{Q}_{\operatorname{MAx}}$ during exercise; this reflects the kinetics of blood flow, mitochondrial enzyme activation, and changes in cytosolic redox state. The flux through the glycogenolytic pathway is set by the activity of glycogen phosphorylase, whose activity in vivo is largely that of the $a$ form alone, and strongly dependent on the concentration of its substrate $\mathrm{Pi}^{27}$ We use the phosphate concentration and the glycogenolytic rate to calculate the apparent maximum rate of glycogenolysis $\left(\mathrm{L}_{\mathrm{MAX}}\right)$, which is an approximate measure of the phosphorylase $a$ activity at each point. To distinguish between the effects of reductions in effective muscle mass and in oxidative ATP synthesis on the changes in exercise, we calculate the overall ATP costs of work. ${ }^{25}$ The overall nonoxidative cost of work will tend to be increased both by a pathological fall in the oxidative contribution (so the nonoxidative contribution must rise) and by a decrease in effective muscle mass (which requires increased ATP supply by all means). The overall oxidative cost of work will tend to be decreased by a fall in the oxidative contribu- tion to exercise, but increased by a fall in effective muscle mass. Thus falls in effective muscle mass and oxidative contribution have the same effects on the nonoxidative cost of work, but opposite effects on the oxidative cost of work. Furthermore, the nonoxidative cost of work per unit effective muscle mass will tend to be increased by a fall in the oxidative contribution to exercise, while the oxidative cost of work per unit effective muscle mass will tend to be decreased. ${ }^{25}$

Results are presented as mean (SE). Statistical significance of differences were determined by factorial or repeated-measures analysis of variance or Student's paired or unpaired $t$ test, as appropriate. Correlations were analysed using Spearman's coefficient.

\section{Results}

Calculated from the kinetics of recovery from exercise, $Q_{\text {MAx }}$ was decreased in both muscles in congestive heart failure compared with control values. A corresponding increase in the phosphocreatine recovery halftime was significant in the flexor digitorum superficialis only (table).

During exercise, in the flexor digitorum superficialis (table) the initial ATP synthesis rate was increased by $73 \%$ in congestive heart failure suggesting a $40 \%$ decrease in effective muscle mass. Throughout exercise, $\mathrm{pH}$ and $\mathrm{PCr} /(\mathrm{PCr}+\mathrm{Pi})$ were reduced and ADP concentration was increased compared with the controls (fig 1), although no significant difference could be demonstrated in the end-exercise values alone (table). Exercise duration 
${ }^{31} \mathrm{P}$ magnetic resonance spectroscopy data from forearm and calf muscle in patients with congestive heart failure and controls (mean (SE))

\begin{tabular}{|c|c|c|c|c|c|c|}
\hline \multirow[b]{2}{*}{ Quantity (Unit) } & \multicolumn{3}{|c|}{ Forearm muscle } & \multicolumn{3}{|l|}{ Calf muscle } \\
\hline & Controls & All $C H F$ & Trained & Controls & All $C H F$ & Trained \\
\hline \multicolumn{7}{|c|}{ Analysis of exercise response } \\
\hline Effective muscle mass (arbitrary units) & $9(1)$ & $5(1)^{\star}$ & $6(1)^{\star}$ & $13(2)$ & $5(1)^{\star}$ & $10(2) \dagger$ \\
\hline $\begin{array}{l}\text { Duration (min) } \\
\text { End exercise: }\end{array}$ & $14 \cdot 2(0 \cdot 2)$ & $11(1)^{\star}$ & $12(1)^{\star} \dagger$ & $16(1)$ & $9(1)^{\star}$ & $9(1)^{\star} \dagger$ \\
\hline $\begin{array}{l}\text { End exercise: } \\
\text { pH }\end{array}$ & \multicolumn{2}{|c|}{ End exercise: } & $6.49(0.06)$ & $6.83(0.04)$ & $6.79(0.05)$ & $6.88(0.05)$ \\
\hline $\mathrm{PCr} /(\mathrm{PCr}+\mathrm{Pi})$ & $0.38(0.08)$ & $0.35(0.03)$ & $0.28(0.03)$ & $0.51(0.04)$ & $0.42(0.04)$ & $0.59(0.05) \dagger$ \\
\hline$[\mathrm{ADP}](\mu \mathrm{M})$ & $32(4)$ & $45(8)$ & $58(17)$ & $38(4)$ & $68(12)^{\star}+t$ & $32(4) \dagger$ \\
\hline \multicolumn{7}{|l|}{ Overall ATP cost of work: } \\
\hline Nonoxidative $(\mathrm{mM})$ & $4(1)$ & $10(2)^{\star}$ & $8(3)^{\star} \dagger$ & $2(1)$ & $6(1)^{\star}$ & $3(1) \dagger$ \\
\hline Oxidative (mM) & $9(1)$ & $9(1)$ & $10(2)$ & $6(1)$ & $17(4)^{\star}$ & $8(1)^{\star}$ \\
\hline \multicolumn{7}{|l|}{$\begin{array}{l}\text { ATP cost of work per unit effective } \\
\text { muscle mass: }\end{array}$} \\
\hline Nonoxidative (mM) & $0 \cdot 3(0 \cdot 1)$ & $0 \cdot 4(0 \cdot 1)$ & $0.4(0 \cdot 1) \dagger$ & $0 \cdot 3(0 \cdot 1)$ & $0 \cdot 3(0 \cdot 1)$ & $0 \cdot 2(0 \cdot 1)$ \\
\hline Oxidative (mM) & $0 \cdot 8(0 \cdot 1)$ & $0 \cdot 6(0 \cdot 1)^{\star}$ & $0 \cdot 8(0 \cdot 2)$ & $0 \cdot 7(0 \cdot 1)$ & $0 \cdot 7(0 \cdot 1)$ & $0 \cdot 8(0 \cdot 1)$ \\
\hline \multicolumn{7}{|c|}{ Mitochondrial function analysed in recovery from exercise } \\
\hline $\begin{array}{l}\text { PCr halftime (min) } \\
\mathrm{Q}_{\operatorname{MAX}}(\mathrm{mM} / \min )\end{array}$ & $\begin{array}{l}0 \cdot 8(0 \cdot 1) \\
51(4)\end{array}$ & $\begin{array}{l}1 \cdot 0(0 \cdot 1)^{\star} \\
36(2)^{\star}\end{array}$ & $\begin{array}{l}0 \cdot 7(0 \cdot 1) \\
49(8) \dagger\end{array}$ & $\begin{array}{l}0 \cdot 6(0 \cdot 1) \\
39(3)\end{array}$ & $\begin{array}{l}0 \cdot 8(0 \cdot 1) \\
29(4)^{\star}\end{array}$ & $\begin{array}{l}0 \cdot 6(0 \cdot 2) \dagger \\
33(6) \dagger\end{array}$ \\
\hline \multicolumn{7}{|c|}{ Proton efflux analysed in recovery from exercise } \\
\hline Initial rate $(\mathrm{mM} / \mathrm{min})$ & $13(1)$ & $6(1)^{\star}$ & $11(2)^{\star}$ & $2(1)$ & $5(1)$ & $3(1)$ \\
\hline Rate constant $(\mathrm{mM} / \mathrm{min}) / \mathrm{pH}$ unit & $30(3)$ & $13(2)$ & $28(7) \dagger$ & $28(18)$ & $29(7)$ & $26(11)$ \\
\hline
\end{tabular}

was reduced. Taking account of the reduced work, the overall (whole-exercise) nonoxidative ATP cost of work was increased by $150 \%$, most of the abnormality being in the glycogenolytic component. Taking account of the reduced effective muscle mass, the nonoxidative cost of work per unit effective muscle mass was increased by $38 \%$ in the congestive heart failure group, while the oxidative cost of work per unit effective muscle mass was reduced by $26 \%$.

Results in gastrocnemius (table) were similar. Initial ATP synthesis rate was increased by $88 \%$ compared with the controls, implying a $50 \%$ decrease in effective muscle mass. Throughout exercise, $\mathrm{pH}$ and $\mathrm{PCr} /(\mathrm{PCr}+\mathrm{Pi})$ were reduced and ADP concentration was increased compared with controls $(P<0.05)$;

Figure 2 ATP synthesis rates during exercise in flexor digitorum superficialis. The figure shows the rates of $A T P$ synthesis by $(A)$ net phosphocreatine depletion, (B) glycogenolysis, and (C) oxidation during 8 exercise spectra in patients (closed circles) and controls (open circles). (Oxidation rate is not shown for the first exercise spectrum, where it is difficult to establish precisely.) Results were significantly different from control $(P<0.05)$ for phosphocreatine depletion and glycogenolytic ATP synthesis but not for oxidative ATP synthesis $(P=0.7)$. All results are given as mean (SE).
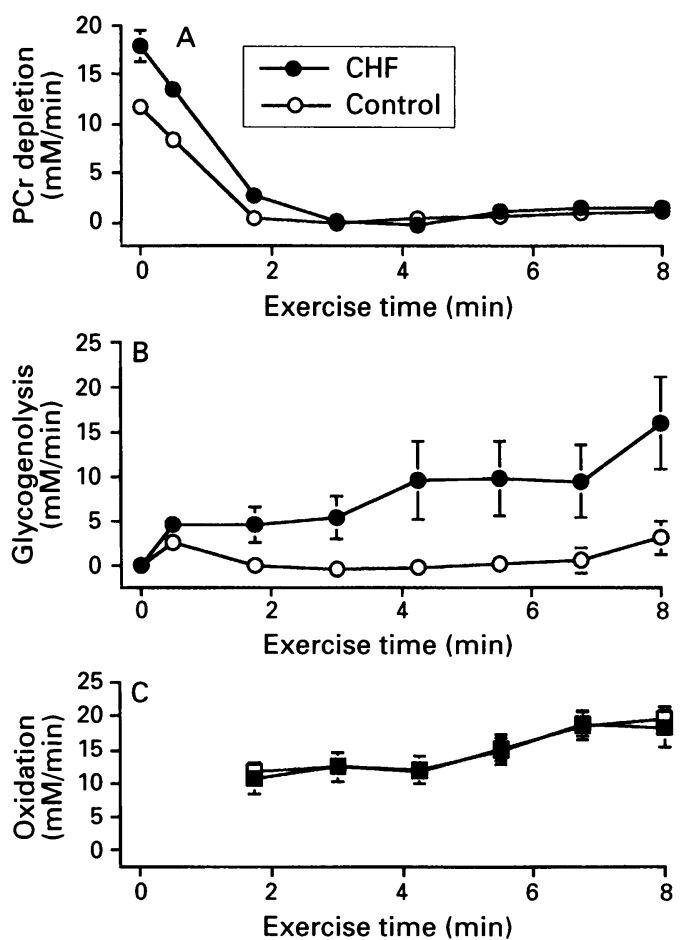

end-exercise $\mathrm{pH}$ and $\mathrm{PCr} /(\mathrm{PCr}+\mathrm{Pi})$ were not significantly different from controls, although end-exercise ADP concentration was increased. Exercise duration was reduced, and correlated with initial ATP synthesis rate $(P<0.04)$, which suggests that reduced effective muscle mass is a substantial contributor to exercise intolerance. The overall nonoxidative cost of work was increased by $210 \%$, and the overall oxidative cost of work by $190 \%$. Taking account of the reduced effective muscle mass, the overall nonoxidative and oxidative costs of work per unit effective muscle mass were not significantly abnormal.

In flexor digitorum superficialis it is possible to establish that NYHA functional class had no significant influence on $\mathrm{Q}_{\mathrm{MAX}}$, proton efflux rate constant, effective muscle mass, and overall nonoxidative cost of work. Only in classes III and IV taken together was the oxidative cost of work per unit effective muscle mass significantly reduced $(P<0.05)$. No difference in results between patients with cardiac failure secondary to coronary artery disease $(n=18)$ and those with idiopathic cardiomyopathy $(n=6)$ was detected.

In both flexor digitorum superficialis and gastrocnemius the main effect of training was to increase $\mathrm{Q}_{\max }$ to a normal value (table). Effective muscle mass in gastrocnemius was also increased by training, although in flexor digitorum superficialis there was no significant difference. In both muscles, duration and the overall nonoxidative cost of work were improved by training; taking account of changes in effective muscle mass, the nonoxidative cost of work per unit effective muscle mass was decreased in flexor digitorum superficialis but not gastrocnemius.

Results from flexor digitorum superficialis were used to calculate rates of ATP turnover during exercise (fig 2). In controls, rates of glycogenolysis and phosphocreatine depletion were small after an initial burst (fig $2 \mathrm{~A}$ and B), and the oxidative ATP synthesis rate rose (fig 

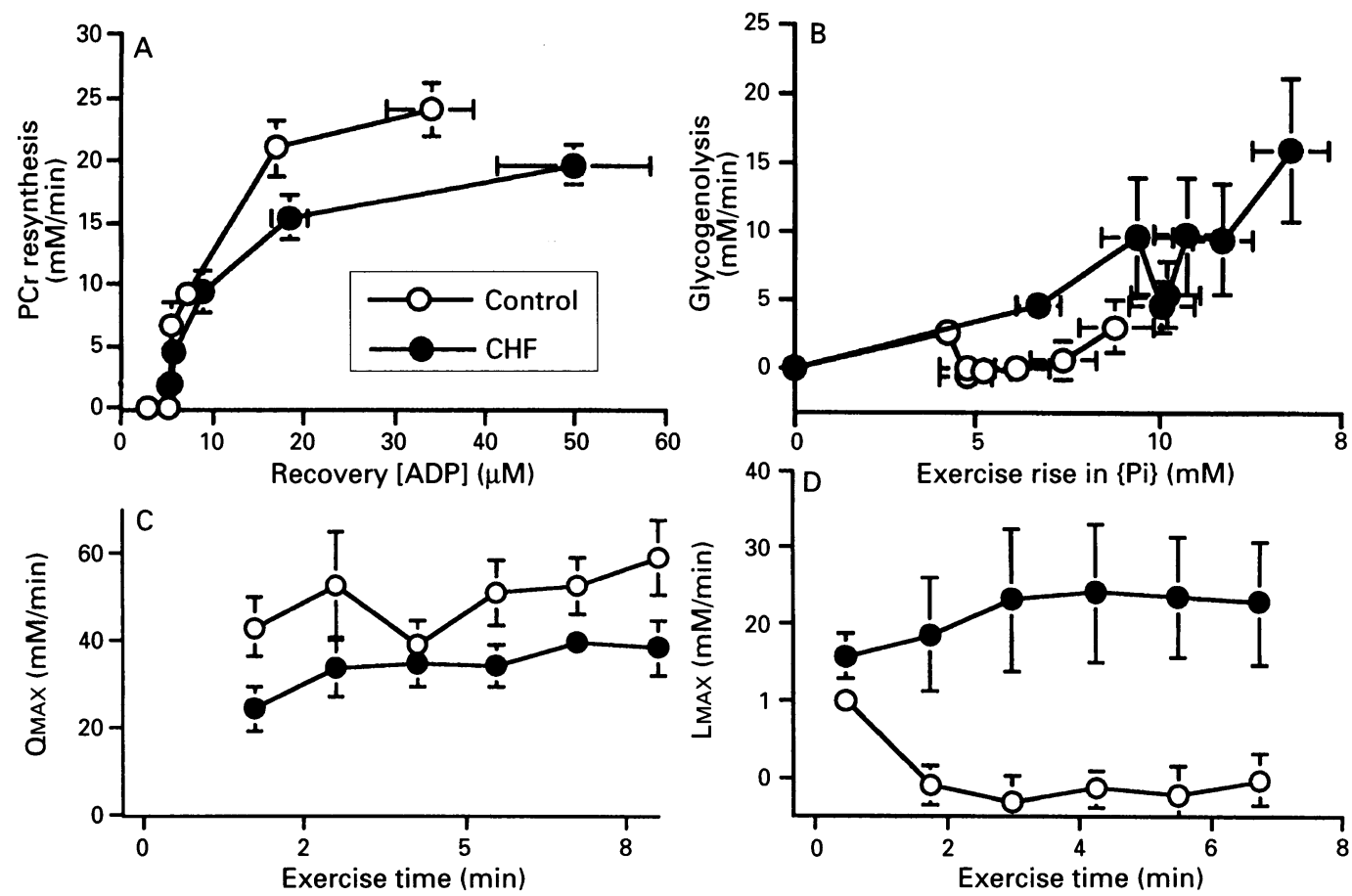

Figure 3 Some metabolic relations in flexor digitorum superficialis of patients (closed circles) and controls (open circles). (A) The rate of phosphocreatine resynthesis during the first 5 min of recovery from exercise, plotted as a function of $A D P$ concentration. (B) The rate of glycogenolytic ATP synthesis during the first 8 exercise spectra, plotted as a function of the increase in inorganic phosphate concentration above basal. (C) The apparent maximum rate of oxidative ATP synthesis $\left(Q_{M A X}\right)$ during exercise, calculated from the rate of oxidative ATP synthesis and the ADP concentration (value not shown for the first exercise spectrum, where it is difficult to establish precisely). (D) The apparent maximum rate of glycogenolysis $\left(L_{M A X}\right)$ during exercise, an approximate measure of the activity of glycogen phosphorylase as calculated from the rate of glycogenolytic ATP synthesis and the inorganic phosphate concentration; results were significantly different from control $(P<0.05)$. All results are given as mean $(S E)$.

2C) with ADP concentration (fig 1C). In patients the initial rates of phosphocreatine depletion rate and glycogenolysis were increased compared with controls, although there was little difference in oxidative ATP synthesis rate (fig 2C). Calculated net proton efflux was increased in congestive heart failure $(P<0.002$, not shown), because of the greater intracellular acidification. Extending the analysis, apparent $Q_{\operatorname{mAx}}$ during exercise was approximately constant with time and lower in congestive heart failure than in controls (fig 3C). The rate of glycogenolysis had an approximately linear dependence on the concentration of inorganic phosphate, a substrate of glycogen phosphorylase (fig 3B). This relation was normal in congestive heart failure for the initial burst of glycogenolysis (the leftmost point in fig 3B), despite the larger absolute values of glycogenolytic rate and phosphate concentration, while during subsequent exercise the glycogenolytic rate was high in relation to the phosphate concentration. This analysis can be taken further by calculating the effective maximum rate of glycogenolysis $\left(\mathrm{L}_{\mathrm{MAX}}\right)$, which reflects the activity of glycogen phosphorylase $a$ during exercise (fig 3D). In controls this decreased to almost zero after the initial burst, increasing later as the power output became large. Initially, $\mathrm{L}_{\mathrm{MAx}}$ was not significantly abnormal (16 (3) $\mathrm{mM} / \mathrm{min}$ in congestive heart failure $v 10$ (2) $\mathrm{mM} / \mathrm{min}$ in controls), but in contrast to controls it remained high throughout exercise. By the end of exercise, both groups showed similar rates of oxidative and nonoxidative ATP synthesis and similar values of $\mathrm{L}_{\mathrm{MAx}}$ (42 (9) $\mathrm{mM} / \mathrm{min}$ in congestive heart failure $v 46$ (8) $\mathrm{mM} / \mathrm{min}$ in controls).

\section{Discussion}

Reduced net proton efflux during exercise, reduced muscle mass and/or metabolic efficiency, reduced oxidative ATP synthesis rate, and over-activation of glycogenolysis could all contribute to the exercise abnormalities. ${ }^{8}$ The overall time-course of $\mathrm{pH}$ recovery was normal (fig 1D), although a detailed analysis of the start of recovery $(t=0-0.8 \mathrm{~min})$ suggests that in patients the rate and rate constant of proton efflux were both slightly decreased (table), possibly because of reduced vascular proton washout or a decrease in activity of sodiumlinked processes of proton efflux. ${ }^{8}$ However, there was no large abnormality of proton efflux as is seen in peripheral vascular disease. ${ }^{26}$ Thus although proton efflux rates during exercise cannot be assessed directly, the present analysis suggests that they were increased in congestive heart failure, due to the larger changes in cell $\mathrm{pH}$; reduced efflux was therefore not a cause of intracellular acidification.

Alterations in muscle mass and/or metabolic efficiency can be established either from rates of ATP synthesis in ischaemic exercise ${ }^{19}$ or from the start of aerobic exercise, when oxidative ATP synthesis makes a negligible contribution. ${ }^{28}$ In the present work, the initial rate of ATP synthesis was increased in both 
muscles, suggesting a $50-65 \%$ decrease in effective muscle mass (table), with no relation to functional class. Part of this abnormality could be due to muscle fibre atrophy. ${ }^{20}$ However, imaging studies reveal only 15-20\% atrophy in human gastrocnemius in congestive heart failure. ${ }^{2}$ The $30 \%$ decrease in maximum voluntary contraction in forearm muscle in congestive heart failure ${ }^{19}{ }^{29}$ probably represents an upper limit on the amount of structural atrophy. A study of ischaemic forearm exercise using a workload scaled to maximum voluntary contraction showed a $50 \%$ increase in total ATP consumption rate in congestive heart failure, ${ }^{19}$ which is equivalent to at least a $30 \%$ decrease in metabolic efficiency. In view of this, it seems likely that gross muscle atrophy is unlikely to be able to account for the increased changes during exercise in congestive heart failure, which suggests that an appreciable part of the effect observed here must be due to a loss of intrinsic metabolic efficiency. This might be associated with fibre type changes. ${ }^{19}$ As we discuss below, this defect dominates the abnormalities in the response to the later stages of exercise.

A reduced capacity for oxidative ATP synthesis can be established from the hyperbolic relation between phosphocreatine resynthesis rate $(\approx$ oxidative ATP synthesis rate) and its driving force, ADP concentration (fig 3A) ${ }^{8}$ This suggests a defect in the maximum rate of oxidative ATP synthesis of about $30 \%$ in both muscles (table), with no relation to functional class. The same conclusion was arrived at in a different way in a study of phosphocreatine recovery in gastrocnemius, ${ }^{2}$ and is consistent with the $30-60 \%$ defect in maximum oxygen uptake in gastrocnemius. ${ }^{11}$ Both reduced mitochondrial content and abnormalities of blood flow may contribute to the observed abnormalities (see Introduction).

Regardless of its cause, a reduction in muscle mass or efficiency would increase the required rate of ATP synthesis by all pathways, while reduction in oxidative capacity tends to reduce the contribution of oxidative ATP synthesis. In the event, oxidative ATP synthesis rates in flexor digitorum superficialis were similar in congestive heart failure both during exercise (fig 2C), because the decreased oxidative capacity was largely compensated for by the increased ADP concentration (fig 1C). The same was true in gastrocnemius (table). (This increase in ADP concentration is implicit in the increase in the $\mathrm{Pi} / \mathrm{PCr}$ ratio reported in exercising forearm muscle ${ }^{14}$ and gastrocnemius $^{2}$ in congestive heart failure.)

The defects in maximum rate of oxidative ATP synthesis and in effective muscle mass both contribute to the increased nonoxidative cost of work (which correlated with effective muscle mass in both muscles, $P<0.05$ ). In both muscles the nonoxidative cost of work per unit of effective muscle mass is normal (table). In addition, in flexor digitorum superficialis the oxidative cost of work per unit effective muscle mass was decreased by $26 \%$ (table), suggesting that despite the increased ADP concentration, the overall contribution of oxidative ATP synthesis was reduced. Thus the abnormalities in flexor digitorum superficialis in congestive heart failure patients arise more from the higher ATP synthesis rate required because of decreased mass and/or efficiency, than from the oxidative defect, which is largely compensated by an increase in $\mathrm{ADP}$ concentration. The situation in gastrocnemius is similar, except that the oxidative defect is swamped by the effects of reduced effective muscle mass, so that the oxidative cost of work is increased despite the oxidative defect.

Rates of glycogenolysis were increased in congestive heart failure, consistent with biopsy evidence, ${ }^{30}$ both in absolute terms (fig $2 \mathrm{~B}$ ) and in relation to the concentration of inorganic phosphate (fig 3B), a substrate for glycogen phosphorylase. We analysed this further by calculating an approximate measure of the activity of phosphorylase $a$ ( $\left.\mathrm{L}_{\text {MAX }}\right)$, which is higher in patients with congestive heart failure than in controls (fig 3D). This response tells us about the metabolic priorities of the muscle in heart failure. If more of the oxidative shortfall were met by phosphocreatine breakdown, or if proton efflux were sufficiently increased, as in human mitochondrial myopathy, ${ }^{31}$ the ADP concentration could rise higher, and so further increase the drive to the defective mitochondrial function. This apparent difference in metabolic control may be associated with the increase in type IIb fibres, ${ }^{32}$ or with possible inadequacies of blood flow, or may simply be a response to the increase in total ATP synthesis rate resulting from decreased metabolic efficiency.

Lastly, the present analysis provides a new perspective on training. In both muscles, training reduced the nonoxidative cost of work (by about $40 \%$ ) (table). In flexor digitorum superficialis, training had only a small effect on the initial ATP synthesis rate and therefore on the effective muscle mass, and so the improvement was mainly due to the increase in $\mathbf{Q}_{\max }$ noted before, ${ }^{22}$ which may be due partly to increases in local blood flow ${ }^{33} 34$ and partly to increases in mitochondrial content. ${ }^{35}$ In gastrocnemius, as well as the improvement in $\mathrm{Q}_{\text {MAX }}$ noted before, ${ }^{9}$ the initial ATP synthesis rate was also reduced to near-normal values. The nonoxidative and oxidative costs of work were both improved, and there was no effect on the costs of work per unit effective muscle mass. This suggests that in gastrocnemius, the effect of training on effective muscle mass is dominant. These differences in these training responses may perhaps arise because the leg training protocol used whole body exercise, ${ }^{9}$ while the forearm training was purely local. ${ }^{22}$

This work was supported in part by the Department of Veterans Affairs (JRS), the British Heart Foundation and the Medical Research Council of Great Britain.

1 Arnolda L, Conway M, Dolecki M, Sharif H, Rajagopalan B, Ledingham JGG, et al. Skeletal muscle metabolism in B, Ledingham JGG, et al. Skeletal muscle metabolism in heart failure: a ${ }^{31} \mathrm{P}$ nuclear magnetic resonance spec-

troscopy study of leg muscle. Clin Sci 1990;79:583-9. McCully KK, Mullen JL, et al. Contribution of skeletal muscle atrophy to exercise intolerance and altered muscle metabolism in heart failure. Circulation 1992;85: 1364-73. 
3 Chati Z, Zannad F, Robin-Lherbier B, Escanye JM Jeandel C, Robert J, et al. Contribution of specific skeletal muscle metabolic abnormalities to limitation of exercise capacity in patients with chronic heart failure: a phosphocapacity in patients with chronic heart failure: a phosphorus 31 nuclear

4 Massie B, Conway M, Yonge R, Frostick S, Sleight $P$, Ledingham JGG, et al. Skeletal muscle metabolism in patients with congestive heart failure: relation to clinica severity and blood flow. Circulation 1987;76:1009-19.

5 Rajagopalan B, Conway MA, Massie B, Radda GK Alterations of skeletal muscle metabolism in human studied by phosphorus 31 magnetic resonance spectroscopy in con

6 Arnolda L, Brosnan J, Rajagopalan B, Radda GK. Skeleta muscle metabolism in heart failure in rats. Am $\mathcal{F}$ Physio 1991;261:H434-H442.

7 Thompson CH, Kemp GJ, Rajagopalan B, Radda GK Abnormal ATP turnover in rat leg muscle during exercise and recovery following myocardial infarction. Cardiovas Res 1995;29:344-9.

8 Kemp GJ, Radda GK. Quantitative interpretation of bioenergetic data from ${ }^{31} \mathrm{P}$ and ${ }^{1} \mathrm{H}$ magnetic resonance spectroscopic studies of skeletal muscle: an analytical review. scopic studies of skeletal muscle:
Magn Reson Quart 1994;10:43-63.

9 Adamopoulos S, Coats AJS, Brunotte F, Arnolda L, Meyer $\mathrm{T}$, Thompson $\mathrm{CH}$, et al. Physical training improves $\mathrm{T}$, Thompson $\mathrm{CH}$, et al. Physical training improves
skeletal muscle metabolism in patients with chronic heart skeletal muscle metabolism in patients with
failure. $\mathcal{A m}$ Coll Cardiol 1993;21:1101-6.

10 Thompson CH, Kemp GJ, Sanderson AL, Rajagopalan B, Radda GK. Metabolic abnormalities in skeletal muscle following myocardial infarction in the rat. Clin Sci 1994

11 Wilson JR, Martin JL, Schwartz D, Ferraro N. Exercise intolerance in patients with chronic heart failure: role of impaired nutritive flow to skeletal muscle. Circulation 1984;69:1079-87.

12 Musch TI, Terrell JA. Skeletal muscle blood flow abnormalities in rats with a chronic myocardial infarction: res and exercise. Am F Physiol 1992;262:H41 1-H419.

13 Muller AF, Batin P, Evans S, Hawkins M, Cowley AJ. Regional blood flow in chronic heart failure: the reason for the lack of correlation between patients' exercise tolerance and cardiac output? Br Heart $\mathcal{f} 1992 ; 67: 478-81$.

14 Wiener DH, Fink LI, Maris J, Jones RA, Chance B, Wilson JR. Abnormal skeletal muscle bioenergetics during exercise in patients with heart failure: role of reduced muscle blood flow. Circulation 1986;73:1127-36.

15 Mancini DM, Wilson JR, Bolinger L, Li H, Kendrick K Chance $\mathrm{B}$, et al. In vivo magnetic resonance spectroscopy measurement of deoxymyoglobin during exercise in patients with heart failure. Demonstration of abnormal muscle metabolism despite adequate oxygenation. muscle metabolism desp

16 Matsui S, Tamura N, Hirakawa T, Kobayashi $S$ Takekoshi N, Murakami E. Assessment of working skeletal muscle oxygenation in patients with chronic heart failure. Am Hean 1995 ; $129: 690$

17 Sullivan M, Green H, Cobb F. Skeletal muscle biochemistry and histology in ambulatory patients with long-term heart failure. Circulation 1990;81:518-27.

18 Minotti JR, Christoph I, Oka R, Weiner MW, Wells L Massie BM. Impaired skeletal muscle function in patients with congestive heart failure. Relationship to systemic exercise performance. F Clin Invest 1991;88: 2077-82.
19 Massie BM, Conway M, Rajagopalan B, Yonge R, Frostick $\mathrm{S}$, Ledingham JGG, et al. Skeletal muscle metabolism during exercise under ischaemic condition

20 Wilson JR, Coyle EF, Osbaken M. Effect of heart failure on skeletal muscle in dogs. Am $f$ Physiol 1992;262:H993H998.

21 Mancini B, Coyle E, Coggin A, Beltz J, Ferraro N, Montain S, et al. Contribution of intrinsic skeletal muscle changes to ${ }^{31} \mathrm{P}$ NMR skeletal muscle metabolic abnormalities in patients with chronic heart failure. Circulation 1989;80:1338-46.

22 Stratton JR, Dunn JF, Adamopoulos S, Kemp GJ, Coat AJS, Rajagopalan B. Training partially reverses skeletal muscle metabolic abnormalities during exercise in heart failure. $\mathcal{F}$ Appl Physiol 1994;76:1575-82

23 Stratton JR, Kemp GJ, Daly RC, Yacoub M, Rajagopalan B. Effect of cardiac transplantation on bioenergetic abnormalities of skeletal muscle in congestive heart failure. Circulation 1994;89:1624-31.

24 Arnold DL, Matthews PM, Radda GK. Metabolic recovery after exercise and the assessment of mitochondrial function in vivo in human skeletal muscle by means of P-31 NMR. Mag Reson Med 1984;1:307-15.

25 Kemp GJ, Thompson CH, Taylor DJ, Radda GK ATP production and mechanical work in exercising skeletal muscle: a theoretical analysis applied to ${ }^{31} \mathrm{P}$ magnetic resmuscle: a theoretical analysis applied to ${ }^{31}$ magnetic resonance spectroscopic studies of dialyse
patients. Magn Reson Med 1995;33:601-9.

26 Kemp GJ, Hands LJ, Ramaswami G, Taylor DJ, Nicolaides A, Radda GK. Calf muscle mitochondrial and glycogenolytic ATP synthesis in patients with claudication due to peripheral vascular disease analysed using ${ }^{31} \mathrm{P} \mathrm{mag}$ netic resonance spectroscopy. Clin Sci 1995;89:581-90.

27 Chasiotis D. The regulation of glycogen phosphorylase and glycogen breakdown in human skeletal muscle. Acta Physiol Scand 1983;suppl 518:1-68.

28 Kemp GJ, Thompson CH, Barnes PRJ, Radda GK Comparisons of ATP turnover in human muscle during ischaemic and aerobic exercise using ${ }^{31} \mathrm{P}$ magnetic resonance spectroscopy. Magn Reson Med 1994;31:248-58.

29 Mancini DM, Ferraro N, Tuchler M, Chance B, Wilson JR. Detection of abnormal calf muscle metabolism in patients with heart failure using phosphorus-32 magnetic resonance. Am $\exists$ Cardiol 1988;62:1234-40.

30 Musch TI, Ghaul MR, Tranchitella V, Zelis R. Skeletal muscle glycogen during submaximal exercise in rats with
chronic heart failure. Basic Res Cardiol 1990;85:606-18.

31 Kemp GJ, Taylor DJ, Styles P, Radda GK. The production, buffering and efflux of protons in human skeletal muscle during exercise and recovery. NMR in Biomed 1993;6:73-83.

32 Massie BM, Conway M, Yonge R, Frostick SP, Sleight $P$ Ledingham JGG, et al. ${ }^{31} \mathrm{P}$ nuclear magnetic resonance evidence of abnormal skeletal muscle function in patient with congestive heart failure. Am $f$ Cardiol 1987;60 1309-15.

33 Musch TI, Nguyen CT, Pham HV, Moore RL. Training effects on the regional blood flow response to exercise in myocardial infarcted rats. Am $\mathcal{F}$ Physiol 1992;262:H1846H1852.

34 Minotti JR, Johnson EC, Hudson TL, Zuroske G, Murata G, Fukushima E, et al. Skeletal muscle response to exercise training in congestive heart failure. $\mathcal{F}$ Clin Invest 1990;86:751-8

35 Holloszy JO. Adaptation of muscular tissue to training. Prog Cardiovasc Dis 1976;18:445-58. 